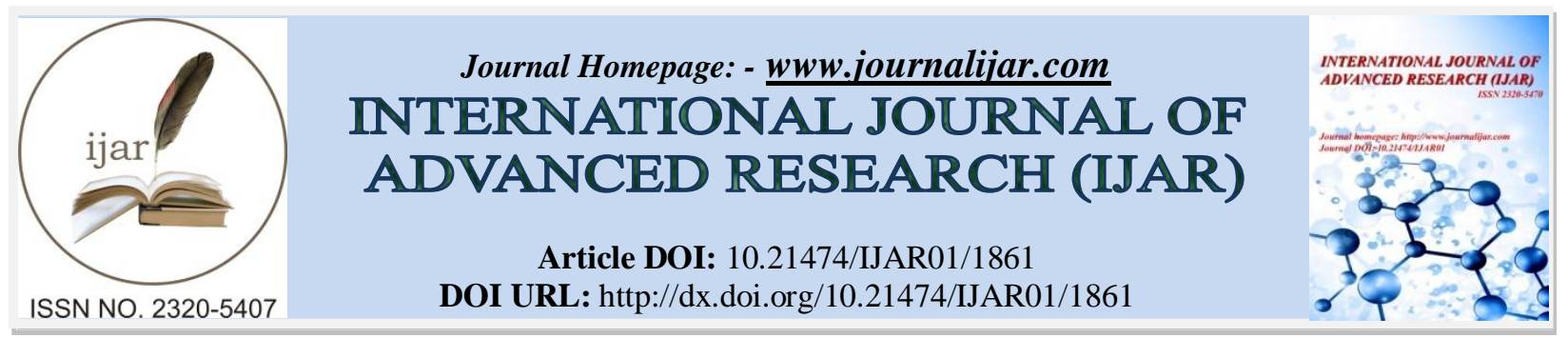

RESEARCH ARTICLE

\title{
METABOLIC SYNDROME VERSUS ITS INDIVIDUAL COMPONENTS AND ANGIOGRAPHIC SEVERITY OF CORONARY ARTERY DISEASE.
}

Hanan I Radwan MD*, Wael Aly Khalil MD and Ahmed Shaker MD.

Department of Cardiology, Faculty of Medicine, Zagazig University, Egypt.

\section{Manuscript Info}

Manuscript History

Received: 12 August 2016

Final Accepted: 22 September 2016

Published: October 2016

Key words:-

Metabolic syndrome, Coronary artery

disease, Gensini risk score, Fasting blood glucose.

\section{Abstract}

Background: Metabolic syndrome (MetS) is a cluster of metabolic traits that confer high risk for coronary artery disease (CAD). This study aimed to assess the relationship between MetS components and severity of CAD .

Methods : Eighty six patients underwent elective coronary angiography, calculation of the metabolic risk score and laboratory evaluation. Patients divided into two groups according to the presence of MetS: group A; 64 patients with $\geq 3$ MetS components and group B; 22 patients with $<3$ components. Assessment of CAD severity using Gensini score.

Results:- There were significant difference between the both groups concerning body mass index, high blood pressure, triglyceride, high density lipoprotein, fasting blood glucose (FBG), serum insulin, insulin resistance and Genseni score $(\mathrm{P}<0.05)$. Significant positive correlation between MetS score and Genseni score $(\mathrm{P}<0.000)$. Multivariate analysis showed that MetS itself did not predict the presence of CAD $(\mathrm{p}=0.49)$, howevere some individual component of MetS show significant predictive value; namely, high FBG > 110 $\mathrm{mg} / \mathrm{dl}$ ( OR 23.748, p<0.001), high TGs > $150 \mathrm{mg} / \mathrm{dl}(\mathrm{OR} 7.35, \mathrm{p}$ $=0.012$ ) and high blood pressure ( OR 6.07, p=0.02).

Conclusion: - Fasting blood glucose, high triglyceride and blood pressure were independent predictors $\mathrm{CAD}$ rather than metabolic syndrome itself. FBG was the most independent factor that predicts CAD.

Copy Right, IJAR, 2016,. All rights reserved.

\section{Introduction:-}

Metabolic syndrome (MetS) remains a heterogeneous disorder with multiple factors that commonly cluster together. This heterogeneity creates a challenge for its definition. [1] Different diagnostic criteria were ideintified; World Health Organization (WHO), National Cholesterol Education Program Adult Treatment Panel III (NCEP/ATP III), and the International Diabetes Federation (IDF). Each organization defines MetS and its required components somewhat differently, suggesting that estimates of MetS prevalence in the same population could differ according to the MetS definition used. [2] MetS according to NCEP/ATP III; refers to a constellation of five risk factors. These include out-of-range waist circumference [for women $>35$ inches $(89 \mathrm{~cm})$; men $>40$ inches $(102 \mathrm{~cm})$ ], triglyceride levels above $150 \mathrm{mg} / \mathrm{dL}$, low high-density lipoprotein (HDL) cholesterol levels [women <50mg/dL; men <40mg/dL, high blood pressure [ $>130 / 85 \mathrm{mmHg}$, and an elevated fasting blood sugar level [>100 $\mathrm{mg} / \mathrm{dL}]$. An individual with at least three of these risk factors qualifies as having MetS .[3] Patients with MetS have an estimated relative risk (RR) 
of 2.35 (2.02-2.73) for CVD, their RR for CVD mortality after adjusting for diabetes and conventional risk factors is estimated 1.39 (1.03-1.86). [4] Even among individuals with angiographically significant coronary artery disease (CAD), the hazard ratio for cardiovascular events is much greater in those with MetS compared with those without .$[5]$

\section{Objective:-}

The study aimed to assess the relationship between MetS components and CAD severity and to determine the most predictive risk factor for $\mathrm{CAD}$ in patients with metabolic syndrome.

\section{Patients and methods:-}

Participants were 86 patients with an evidence of suspected CAD whom were scheduled for elective coronary angiography $48(55.8 \%)$ males and 38(44.2\%) females. Patients with previous PCI, CABG, patients on chronic hemodialysis, and systemic diseases were excluded.

All patients are subjected to full history taking including risk for CAD assessed using standard questionnaires Height and weight were measured. BMI was calculated by the formula, BMI= Body weight $(\mathrm{Kg}) / \mathrm{Height}(\mathrm{m} 2)(\mathrm{The}$ Quetelet Index). [6] Waist circumference was measured in standing subjects midway between the inferior lateral margin of the ribs and the superior lateral border of the iliac crest. Systolic and diastolic blood pressure was measured three times in seated resting subjects by certified staff according to a standard protocol. Hypertension was defined as systolic blood pressure $\geq 140$ and/or diastolic $\geq 90 \mathrm{mmHg}$ or use of antihypertensive medication.

\section{Laboratory work up:-}

Venous sampling was collected in the early morning after an overnight fasting prior to elective coronary angiography for fasting plasma glucose (FPG), Fasting serum triglyceride level (TG), Fasting serum high-density lipoprotein level (HDL), Fasting serum insulin and insulin resistance was evaluated by the homeostatic model assessment $(\mathrm{HOMA}) . \mathrm{HOMA}$ index $=[\{$ fasting insulin $(\mathrm{U} / \mathrm{mL})\} \times\{$ fasting glucose $(\mathrm{mmol} / \mathrm{L})\}] / 22.5 .[7]$

\section{Calculation of metabolic syndrome score:-}

Using NCEP/ATP III, those who had $\geq 3$ of 5 components were classified as having MetS. [3] The patients were divided into two groups according to MetS for each patient.

\section{Coronary angiography:-}

All patients had undergone coronary angiography. Digital coronary angiograms were analyzed offline with an automated edge detection system (Philips Integris 5000, Netherland) and patients with proved CAD were included in the study. Calculation of Gensini score for each patient and percent diameter stenosis (DS) expressed as 25, 50, $75,90,99$, and 100 was scored as $1,2,4,8,16$, and 32 , respectively. Then the score was multiplied by $\times 0.5, \times 1$, $\times 1.5, \times 2.5$, or $\times 5$ according to the segment where the lesion was located. After the score of each lesion was calculated, they were all added together to produce the severity score for the entire coronary system. [8]

\section{Ethics:-}

Informed parental consent was obtained to be eligible for enrollment into the study. The study was done according to the rules of the Local Ethics Committee of Faculty of Medicine, Zagazig University, Egypt.

\section{Statistical analysis:-}

SPSS 20 (SPSS Science, Chicago, IL, USA) for Windows was used for statistical analysis. Continuous variables are presented as mean \pm standard deviation (SD), and categorical variables as percentages. Comparison of categorical and continuous variables between the two groups was performed using chi-square test and independent sample $t$ test, respectively. Linear regression analysis was used to test univariate relations. A P value $<0.05$ was considered statistically significant.

\section{Results:-}

Patients were divided according to the components of MetS into group (A); with $\geq 3$ components of MetS (64 patients, 74.4\%) and group (B) who have < 3 components of MetS (22 patients, 25.6\%). 48 patients were males $(55.8 \%)$ and 38 patients were females (44.2\%). Age of the patients was 55.48 \pm 6.22 years, 31 patients (36\%) were current smokers, 45 patients (52.3\%) were hypertensive, 51 patients $(59.3 \%)$ were diabetic and 44 patients (51.2\%) 
had positive family history of CAD. The weight was $81.14 \pm 12.57 \mathrm{~kg}$ and the height was $1.68 \pm 9.37 \mathrm{~cm}$. Demographic and risk factors for CAD were comparable between both groups with no significant statistical difference regarding age, sex, smoking, height or risk factors for CAD (hypertension, diabetes, and positive family history) (table1).

Table 1:- Demographic data and risk factors in the two study groups.

\begin{tabular}{|c|c|c|c|c|}
\hline \multicolumn{2}{|c|}{ Variable } & $\begin{array}{l}\text { Group }(A) \\
(n=64)\end{array}$ & $\begin{array}{l}\text { Group }(B) \\
(n=22)\end{array}$ & P-Value \\
\hline \multicolumn{2}{|c|}{ Age $X \pm$ SD } & $56.11 \pm 6.45$ & $53.68 \pm 5.20$ & 0.115 \\
\hline \multirow[t]{2}{*}{ Gender } & Female & $32(50 \%)$ & $16(72.7 \%)$ & \multirow[t]{2}{*}{0.133} \\
\hline & Male & $32(50 \%)$ & $6(27.3 \%)$ & \\
\hline \multicolumn{2}{|l|}{ HTN } & $36(56.3 \%)$ & $9(40.9 \%)$ & 0.229 \\
\hline \multicolumn{2}{|l|}{ DM } & $41(64.1 \%)$ & $10(45.5 \%)$ & 0.140 \\
\hline \multicolumn{2}{|c|}{ Smokers } & $20(31.2 \%)$ & $11(50 \%)$ & 0.130 \\
\hline \multicolumn{2}{|c|}{ Family History } & $30(46.9 \%)$ & $14(63.6 \%)$ & 0.220 \\
\hline
\end{tabular}

There was a significant statistical difference between both groups regarding clinical and biochemical characteristics; weight, BMI, high blood pressure, all were significantly higher in-group A ( $<<0.05$ ). (Table 2).

Table 2 :- Clinical Characteristics of both studied groups.

\begin{tabular}{|l|l|l|l|}
\hline \multirow{2}{*}{ Variable X \pm SD } & $\begin{array}{l}\text { Group (A) } \\
(\mathbf{n = 6 4 )}\end{array}$ & $\begin{array}{l}\text { Group (B) } \\
(\mathbf{n = 2 2})\end{array}$ & P-Value \\
\cline { 2 - 4 } & \multicolumn{3}{|l|}{} \\
\hline Height & $168 \pm 10.1$ & $170 \pm 6.65$ & 0.211 \\
\hline Weight & $84.88 \pm 11.22$ & $70.27 \pm 9.78$ & 0.000 \\
\hline BMI & $30.191 \pm 3.3238$ & $24.191 \pm 3.3208$ & 0.000 \\
\hline High BP & $48(75 \%)$ & $11(50 \%)$ & 0.036 \\
\hline
\end{tabular}

Regarding the biochemical variants of MetS; statistically, there was a significant difference between the two groups; TG, HDL, FBG, the fasting serum insulin and HOMA index. They were all significantly higher in group A $(\mathrm{p}<0.05)$ (table 3$)$.

Table 3:- Biochemical characteristics of both studied groups.

\begin{tabular}{|l|l|l|l|}
\hline \multirow{2}{*}{ Variable X +SD } & $\begin{array}{l}\text { Group (A) } \\
\text { (n=64) }\end{array}$ & $\begin{array}{l}\text { Group (B) } \\
(\mathbf{n = 2 2})\end{array}$ & P-Value \\
\cline { 2 - 4 } & \multicolumn{2}{|l|}{0.006} \\
\hline TG & $193.20 \pm 57.49$ & $155.27 \pm 41.63$ & 0.002 \\
\hline HDL & $51.88 \pm 15.33$ & $64.55 \pm 17.89$ & 0.001 \\
\hline fasting serum insulin & $170.80 \pm 75.68$ & $109.05 \pm 61.721$ & 0.014 \\
\hline insulin resistance & $14.73 \pm 10.43$ & $9.74 \pm 6.93$ & 0.014 \\
\hline
\end{tabular}

Regarding the angiographic criteria: In Group (A); 50 patients (78.1\%) had significant CAD while there were 14 patients $(21.9 \%)$ who had normal or non-significant CAD. Ten patients had single vessel disease $(15.6 \%), 21$ patients had two vessels disease $(32.8 \%), 19$ patients had three vessels disease $(29.7 \%)$. Gensini score for this group was $52.65 \pm 38.93$.

In Group (B); there were 6 patients with significant CAD (27.3\%), only 1 of them had single vessel disease (4.5\%), 3 patients had two vessels disease $(13.6 \%)$ and 2 patients had three vessels disease $(9.1 \%)$. There were 16 patients $(72.7 \%)$ with normal or non-significant CAD. Gensini score for this group was $11.93 \pm 21.35$. We demonstrated a significant difference between both groups regarding the presence of CAD and Genseni score $(\mathrm{p}<0.05)($ Table 4$)$. 
Table 4:- Coronary angiographic Characteristics of both studied groups.

\begin{tabular}{|l|l|l|l|}
\hline $\begin{array}{l}\text { Group (A) } \\
(\mathbf{n = 6 4 )}\end{array}$ & $\begin{array}{l}\text { Group (B) } \\
(\mathbf{n = 2 2})\end{array}$ & P-Value \\
\hline Geneni score & $52.65 \pm 38.93$ & $11.93 \pm 21.35$ & 0.000 \\
\hline CAD & $50(78.1 \%)$ & $6(27.3 \%)$ & 0.000 \\
\hline Single vessel disease & $10(15.6 \%)$ & $1(4.5 \%)$ & \\
\hline Two vessels disease & $21(32.8 \%)$ & $3(13.6 \%)$ & \\
\hline Three vessels disease & $19(29.7 \%)$ & $2(9.1 \%)$ & \\
\hline Normal or minimal & $14(21.9 \%)$ & $16(72.7 \%)$ & 0.000 \\
\hline
\end{tabular}

There were statistically significant positive correlation between metabolic syndrome score and Genseni score $(\mathrm{p}<0.05)$ (Table 5, Figure 1).

Table 5:- Correlation between metabolic syndrome score and Genseni score.

\begin{tabular}{|l|l|l|}
\hline & R & P \\
\hline Metabolic syndrome score and Genseni score & 0.499 & 0.000 \\
\hline
\end{tabular}

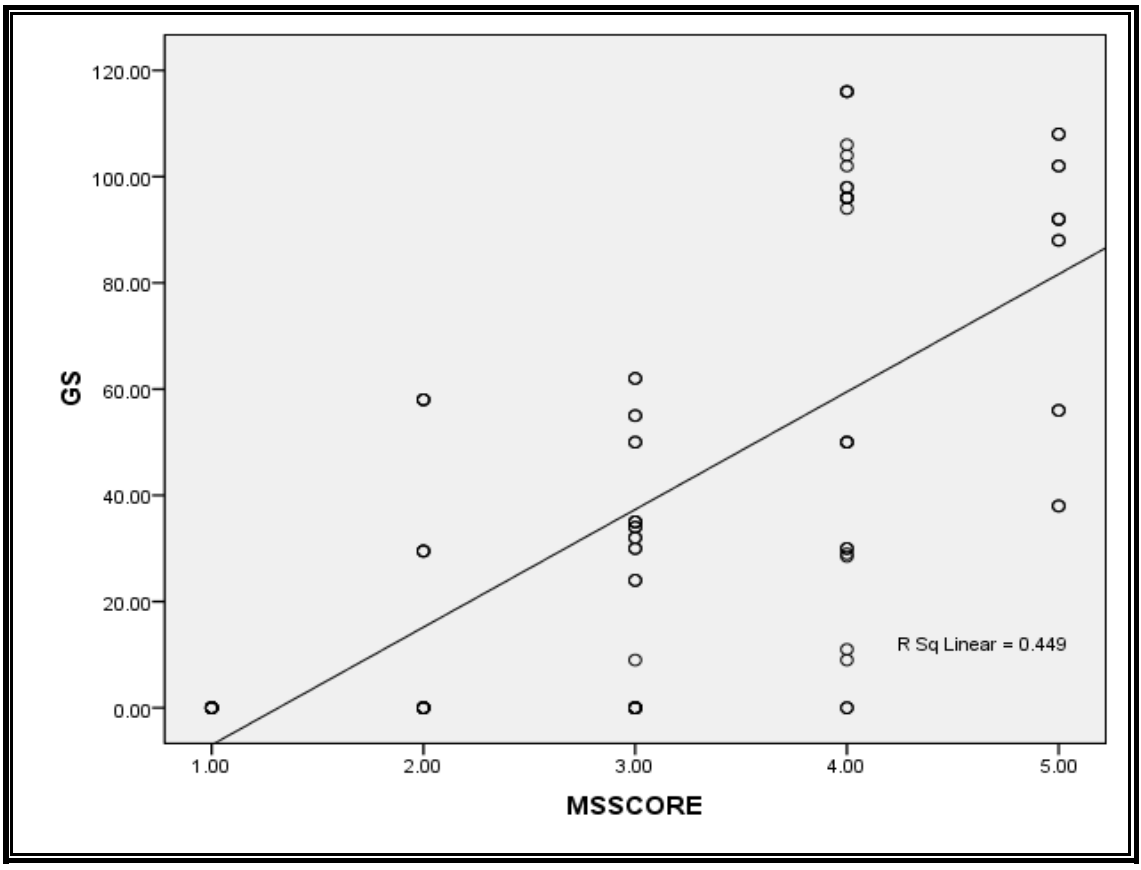

Figure 1:- A scatter diagram is showing significant positive correlation between metabolic syndrome score and Gensini score.

Multivariate regression analysis showed that metabolic syndrome itself did not predict the presence of CAD (OR $0.451, \mathrm{p}=0.489$ ). Instead some individual component of metabolic syndrome show significant predictive value; namely, high FBG more than $110 \mathrm{mg} / \mathrm{dl}(\mathrm{OR} 23.748$,p< 0.001), high TGs > $150 \mathrm{mg} / \mathrm{dl}(\mathrm{OR} 7.349, \mathrm{p}=0.019)$ and high blood pressure $>130 / 85 \mathrm{mmHg}$ ( OR 6.074,p= 0.023). FBG is the most independent factor that predicts CAD. (Table 6).

Table 6:- Multiple clinical and biochemical parameters as determinants of angiographic coronary artery disease in multivariate logistic regression analysis.

\begin{tabular}{|l|c|c|}
\hline Metabolic syndrome component & OR & P. value \\
\hline HDL & 1.379 & 0.692 \\
\hline TG & 7.349 & 0.019 \\
\hline BP & 6.074 & 0.023 \\
\hline FBG & 23.748 & 0.000 \\
\hline BMI & 3.147 & 0.259 \\
\hline MetS & 0.451 & 0.489 \\
\hline
\end{tabular}




\section{Discussion:-}

MetS is defined by a constellation of an interconnected physiological, biochemical, clinical, and metabolic factors that directly increases the risk of atherosclerotic cardiovascular disease and all-causes mortality.[9] In spite being only a cluster of clinical risk factors metabolic syndrome (MetS) has been recognized as independent predictor of cardiovascular diseases ' [10] A handful of previous studies have reported the prevalence of metabolic syndrome among patients with CAD. Although many definitions for metabolic criteria had been published, still it is debatable which component of it is the most predictive for patients' vulnerability to CAD. The current study demonstrated a highly statistically significant difference between both groups regarding body weight and BMI. This results in agreement with Wormser et al. and Flegal et al. [11, 12] Mansour et al. [13] found that BMI was higher in Egyptian patients with both metabolic syndrome and CAD but statistically insignificant. Mohsen Ibrahim et al. [14] explained this discordance to be due to the high prevalence of abdominal obesity in Egyptians.

We found a statistical significant difference between both groups regarding the high blood pressure $>130 / 85 \mathrm{mmHg}$ $(\mathrm{p}=0.036)$. This result agrees with the previous studies provided that high BP is a major and independent cardiovascular risk factor, and hypertension tends to cluster with other metabolic risk factors. [15] Hypertensive individuals tend to have systemic endothelial dysfunction and chronic subclinical inflammation; [16] about half of patients with essential hypertension have insulin resistant. [17] This concludes that hypertension now is increasingly recognized as a powerful risk for cardiac and cerebrovascular events. [18] Many other studies such as Takatoshi et al, showed the clinical importance of the metabolic syndrome itself regardless of hypertension which presence was independent of other potential confounders in multivariate analysis. This further confirms the importance of high blood pressure $>130 / 85 \mathrm{mmHg}$ as strong risk factor of CAD in such cases. [19] Patients with metabolic syndrome ( group A) had high levels of the triglyceride(TG), high density lipoprotein (HDL) and fasting blood glucose (FBG), compared to group B (less than 3 components). These finding were in agreement with Mansour et al. and Kim et al. [13,15]

Mottillo et al. demonstrated that visceral adipose tissue is an active endocrine organ that produces several bioactive derivatives including pro inflammatory and pro thrombotic adipokines, and this change is associated with each individual component of MetS. The close relationships among the different components of the syndrome make it difficult to understand what the hidden exact criminal that initiate the atherosclerotic process. [20]

In our present study, we compare the level of serum insulin and HOMA index (as a marker of insulin resistance) in the two groups. It was found that there was statistical significant difference regarding fasting serum insulin and HOMA index, being significantly higher in group A. Haffner et al. [21] stated that insulin resistance is an independent risk factor for cardiovascular disease; its presence can lead to macrovascular complications before other features of metabolic syndrome.

We identified that not only CAD is highly prevalent in the patients with metabolic syndrome, but also its severity is more in such cases; Genseni score was higher in metabolic syndrome group. In addition, there was a significant positive correlation between Metabolic syndrome score and Genseni risk score. This agrees with Yavuz et al, who confirmed such a relation.[22]

Patients with metabolic syndrome have more extensive coronary lesions and poorer collateral circulation than those without metabolic syndrome. Hadaegh et al, demonstrated that all MetS definitions were associated with the increased risk of CAD [23].Plaques in subjects with MetS harbor more lipid content and more calcification. [24] Other studies found that patients with and without metabolic syndrome did not differ in the prevalence of CAD. [15] These discrepancies may be due to the difference between different metabolic syndrome criterion used, different ethnicity and age of the study population in different studies. These discrepancies confirm the opinion that metabolic syndrome definition may vary according to ethnic background.

A multivariate regression analysis was done and we found that metabolic syndrome itself did not predict the presence of CAD in our cases (OR 0.451, p =0.489). Instead some individual component of metabolic syndrome show significant predictive value; namely, high FBG more than $110 \mathrm{mg} / \mathrm{dl}$ ( OR 23.748,p<0.001), high TGs > 150 $\mathrm{mg} / \mathrm{dl}(\mathrm{OR} 7.349, \mathrm{p}=0.019)$ and high blood pressure $>130 / 85 \mathrm{mmHg}(\mathrm{OR} 6.074, \mathrm{p}=0.023)$.

In our study, FBG, TGs and high blood pressure more than 130/ $85 \mathrm{mmHg}$ are considered independent factors that can predict CAD rather than metabolic syndrome itself. FBG was the most independent factor that can predict CAD. Nearly the same deduction was noted in many studies that tried to reach the exact and most valuable criterion of metabolic syndrome that links to CAD. In the secondary analysis of the prospective West of Scotland Coronary 
Prevention Study, Metabolic syndrome was not a significant predictor of CAD when adjusted for its component factors in a multivariate model. It was suggest that the syndrome itself conveys no greater information than the sum of its component risk factors. [25] In agreement with our work, other study concluded that diabetes and high FBG not the metabolic syndrome itself predicted the severity and extent of CAD. [26] Other studies high lightened the value of blood pressure and stated that elevated blood pressure for CAD events and high FBG for CAD remained as independent predictors in all definitions. [27]

\section{Conclusion:-}

FBG, high TGs and high blood pressure were independent predictors CAD rather than metabolic syndrome itself. FBG was the most independent factor that predicts CAD.

\section{Recommendation:-}

More workout is needed to solve the issue of different definitions of metabolic syndrome concerning the difference in patients ethnic profile as well as clinical and biochemical profile.

\section{Acknowledgment:-}

The authors thank the staff of catheterization laboratory, cardiology department Zagazig University Hospital for their collaboration as well as our patients who participated in the study.

\section{Declaration of interest:-}

The authors declare that there is no conflict of interest.

\section{Funding:-}

This research did not receive any specific grant from any funding agency in the public, commercial or not-for-profit sector.

\section{References:-}

1. Ziki MD, Mani A. Metabolic syndrome: genetic insights into disease pathogenesis. .Curr Opin Lipidol. 2016 Apr; 27(2):162-171. doi: 10.1097/MOL.0000000000000276.

2. Farias DR, Pereira AF, Rosa G. Metabolic syndrome in coronary artery and occlusive vascular disease: a systemic review. Arq Bras Cardiol. 2010; 94:150-178.

3. National Cholesterol Education Program Expert Panel on Detection, Evaluation, and Treatment of High Blood Cholesterol in Adults (Adult Treatment Panel III). Third report of the National Cholesterol Education Program (NCEP) expert panel on detection, evaluation, and treatment of high blood cholesterol in adults (Adult Treatment Panel III). Final report. Circulation.2002; 106: 3143-3421.

4. Daly CA, Hildebrandt P, Bertrand M, Ferrari R, Remme W, Simoons M, Fox KM; EUROPA investigators. Adverse prognosis associated with the metabolic syndrome in established coronary artery disease: data from the EUROPA trial. Heart. 2007 ; 93 (11):1406-1411. Epub 2007 May 31.

5. Sepulveda J, Murray C. The state of global health in 2014. Science 2014; 345:1275-1278.

6. Weigley ES: Adolphe Quetelet (1796-1874): Pioneer anthropometrist. Nutrition Today .1989; 24:12-16.

7. Matthews D R, Hosker J P, Rudenski A S, Naylor B A, Treacher D F, Turner R C. Homeostasis model assessment: insulin resistance and Beta cell function from fasting plasma glucose and insulin concentrations in man. Diabetologia .1985; 28:412-419.

8. Gensini GG .Amore meaningful scoring system for determining the severity of coronary heart disease. Am J Cardiol .1983; 51:606.

9. Schillaci G, Pirro M, Vaudo G, Mannarino M R,Savarese G, Pucci G, Franklin S S. Metabolic Syndrome Is Associated With Aortic Stiffness in Untreated Essential Hypertension. Hypertension.2005; 45: 1078-1082, doi: 10.1161/01.HYP.0000165313.84007.7d.

10. Ajay K Gupta, Bjorn Dahlof, Peter S Sever, Neil R Poulter, and for the Anglo-Scandinavian Cardiac Outcomes Trial-Blood Pressure Lowering Arm (ASCOT-BPLA) investigators. Metabolic syndrome, independent of its components, is a risk factor for stroke and death but not for coronary heart disease among hypertensive patients in the ASCOT-BPLA. Diabetes Care. 2010 Jul; 33(7): 1647-1651. . doi: 10.2337/dc092208.

11. Emerging Risk Factors Collaboration, Wormser D, Kaptoge S, Di Angelantonio E, Wood AM, Pennells L, Thompson A, et al. Separate and combined associations of body-mass index and abdominal adiposity with 
cardiovascular disease: collaborative analysis of 58 prospective studies. Lancet. 2011 Mar 26; 377(9771):10851095. doi: 10.1016/S0140-6736 (11) 60105-0.

12. Flegal KM, Kit BK, Orpana H, Graubard BI. Association of all-cause mortality with overweight and obesity using standard body mass index categories: a systematic review and meta-analysis. JAMA. 2013 Jan 2; 309(1):71-82. doi: 10.1001/jama.2012.113905.

13. Mansour M Mostafa, Abdelaziz R Hassan, Mohammed Abdel Hady, Yasser E. Mohammed, Hesham M. ElZomor .The Correlation Between Metabolic Syndrome and Coronary Artery Disease in Egyptian Patients. New York Science Journal. 2012; 5(12):183-189.

14. Ibrahim MM (2009): Subcutaneous and visceral adipose tissue: structural and functional differences. Obes Rev. 2010 Jan; 11(1):11-18. doi: 10.1111/j.1467-789X.2009.00623.x. Epub 2009 Jul 28.

15. Kim JY, Mun HS, Lee BK, Yoon SB, Choi EY, Min PK et al. Impact of metabolic syndrome and its individual components on the presence and severity of angiographic coronary artery disease. Yonsei Med J. 2010; 51(5):676-682.

16. Festa A, D'Agostino R Jr, Howard G, Mykkänen L, Tracy RP, Haffner SM. Chronic subclinical inflammation as part of the insulin resistance syndrome: the insulin resistance atherosclerosis study (IRAS): Circulation. 2000; 102:42- 47.

17. Lind L, Berne C ,Lithell H. Prevalence of insulin resistance in essential hypertension. J Hypertens.1995 ; 13:1457-1462.

18. Yonetsu T, Kato K, Uemura S, Kim BK, Jang Y, Kang SJ, Park SJ, Lee S, Kim SJ, Jia H, et al . Features of Coronary Plaque in Patients With Metabolic Syndrome and Diabetes Mellitus Assessed by 3-Vessel Optical Coherence Tomography. Circ Cardiovasc Imaging .2013;6:665-673. doi: 10.1161/CIRC IMAGING.113.000345. Epub 2013 Aug 6.

19. Kasai T, Miyauchi K, Kajimoto K, Kubota N, Dohi T, Kurata T, Amano A, Daida H. The adverse prognostic significance of the metabolic syndrome with and without hypertension in patients who underwent complete coronary revascularization. Journal of Hypertension 2009, 27:1017-1024. doi: 10.1097/HJH.0b013e32832961cf.

20. Mottillo S, Filion KB, Genest J, Joseph L, Pilote L, Poirier P, Rinfret S, Schiffrin EL, Eisenberg MJ. The Metabolic Syndrome and Cardiovascular Risk: A Systematic Review and Meta-Analysis. JACC 2010 Sep 28; 56(14):1113-32. doi: 10.1016/j.jacc.2010.05.034.

21. Haffner SM .The metabolic syndrome: inflammation, diabetes mellitus, and cardiovascular disease. Am J Cardiol. 2006 Jan 16; 97(2A):3A-11A.

22. Yavuz B, Kabakci G, Aksoy H, Tulumen E, Deveci O S, Aytemir K, et al. Determining the relationship between metabolic syndrome score and angiographic severity of coronary artery disease. International Journal of Clinical Practice. 2008 ; 62 (5) : 717-722. DOI: 10.1111/j.1742-1241.2008.01702.

23. Hadaegh F, Mohebi R, Cheraghi L, Tohidi M, Moghaddam NB, Bozorogmanesh M, et al. Different metabolic syndrome definitions predict cerebrovascular events and coronary heart disease independent of their components? 9 Years Follow-Up of the Tehran Lipid and Glucose Study. Stroke. 2012; 43:1669-1671.

24. Yonetsu T, Kato K, Uemura S, Kim BK, Jang Y, Kang SJ, et al. Features of coronary plaque in patients with metabolic syndrome and diabetes mellitus assessed by 3-vessel optical coherence tomography . Cardiovascular Imaging. 2013; 6: 665-673.

25. Kahn R ,Buse J , Ferrannini E, Stern M . Metabolic Syndrome: Time for a Critical Appraisal Joint statement from the American Diabetes Association and the European Association for the Study of Diabetes. Diabetes Care. 2005 Sep; 28(9):2289-2304.

26. Srinivasan MP, Kamath PK, Bhat NM, Pai ND, Manjrekar PA, Mahabala C. Factors associated with no apparent coronary artery disease in patients with type 2 diabetes mellitus for more than 10 years of duration: a case control study. Cardiovasc Diabetol. 2015; 14: 146.

27. Qiao Q, Laatikainen T, Zethelius B, Stegmayr B, Eliasson M, Jousilahti P, Tuomilehto J. Comparison of definitions of metabolic syndrome in relation to the risk of developing stroke and coronary heart disease in finnish and Swedish cohort. Stroke. 2009; 40: 337-43. 